\title{
A Tracking-Resistant Pseudonym Scheme for Location Privacy in VANETS
}

\author{
LingLing Wang ${ }^{a}$, LiJun Sun, Min Shao \\ College of Information Science and Technology, Qingdao University of Science and Technology, \\ Qingdao, 266061, China \\ aemail: teacherwll@163.com
}

Keywords: Pseudonym Change; Location Privacy; Domain-Specific Signature

\begin{abstract}
Pseudonym changing is the state-of-the-art approach for resist tracking attack of vehicles in vehicular ad-hoc networks (VANETs). However, most of the proposed pseudonym changing schemes cannot guarantee to resist the tracking attack, namely, provide the unlinkability of vehicles, meanwhile they prefer to generate and store a set of pseudonyms before vehicles' travelling, which causes large storage cost. Based on the dynamic domain-specific pseudonymous signatures, we propose a tracking-resistant pseudonym scheme (TRPS) that provides unlinkability for honest users across domains. Our scheme can protect the location privacy of vehicles and can also trace the dishonest vehicles if some disputed message is broadcast in the wireless channel.
\end{abstract}

\section{Introduction}

Vehicular ad hoc networks (VANETs) are initially designed for enhancing driving safety and convenience in transportation systems. In VANETs, vehicles can communicate with each other and road-side units (RSUs). This enables a range of applications, such as emergence reporting, collision warning, and infotainments, which improve the road safety and better driving experiences.

In many applications, vehicles need to periodically broadcast an authenticated safety message, which includes vehicular status information such as verifiable identity, position, speed, and acceleration. Due to the nature of the wireless communications, an attacker can easily eavesdrop on all the broadcast messages and determine the locations visited by the vehicles over a period of time, which compromise the privacy of drivers ${ }^{[1]}$. Because the lack of privacy may hinder the wide acceptance of VANET technology ${ }^{[2]}$, it is important to protect the location privacy of vehicles in VANETs. A commom approach to avoid privacy attack is the use of pseudonyms instead of static identifiers $^{[3,4]}$. Pseudonyms represent a set of certified public keys related to the vehicle.

Since a pseudonym scheme requires that pseudonyms cannot be linked to each other, pseudonym changing has become a popular approach to achieve unlinkability which enables hiding user identities while enabling access control and the effective protection against sybil attacks. In pseudonym changing schemes, how and when to change pseudonym is the critical problem.

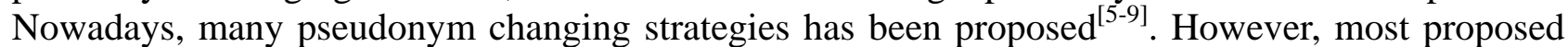
schemes prefer to generate and store a set of pseudonyms in the vehicle's OBU before travelling ${ }^{[4]}$, which cause large storage cost. Moreover, the unlikability of pseudonyms cannot be guaranteed by simple pseudonym change ${ }^{[10]}$.

In this paper, we address to overcome this issue and to facilitate generating pseudonyms and satisfying the required level of privacy protection in VANETs. We propose a tracking-resistant pseudonym scheme (TRPS) for location privacy in VANETS. Our scheme supports efficient and unlinkable pseudonym changes as well as the blacklisting of malicious pseudonym holders.

\section{The Proposed Tracking-Resistant Pseudonym Scheme}

By using the concept of domain-specific pseudonym signatures ${ }^{[11]}$, our proposed TRPS satisfies the properties of seclusiveness, unforgeability and cross-domain anonymity. By adding the tracking algorithm, our TRPS supports the property of traceability of dishonest participants. There are four participants in our scheme, that is, Trusted Third Party (TTP), road-side units (RSUs), vehicle 
member $V_{i}$ who wants to broadcast some message, and the verifiers, who are in different levels of hierarchy when communicating. $V_{i}$ interacts with TTP to obtain his long-term private key. When travelling in region $j, V_{i}$ obtain the corresponding $d p k_{j}$ from nearest $R S U$.

When revocation or tracking is needed, $R S U$ collect the requirements and ask for TTP to revoke the pseudonym or trace the identity of dishonest vehicles. Our TRPS mainly consists of the following eight parts:

(1) $\operatorname{Setup}(\lambda)$ : On a security parameter $\lambda$, TTP chooses an asymmetric bilinear map: $e: G_{1} \times G_{2} \rightarrow G_{T}$, where $G_{1}, G_{2}, G_{T}$ are three multiplicative groups of order $p$, and $G, H$ is a generator of $G_{1}, G_{2}$; $e$ satisfies the following properties: Bilinearity: $e(a G, b H)=e(G, H)^{a b}$; Nondegeneracy: there exists $G \in G_{1}$ and $H \in G_{2}$, such that $e(G, H) \neq 1_{G_{T}}$, where $1_{G_{T}}$ is the identity of $G_{T}$; and Computability: there exists an efficient algorithm to compute $e(G, H)$ for all $G \in G_{1}$ and $H \in G_{2}$.

Choose $P, Q$ as the generator of $G_{1}$, and $H$ as the generator of $G_{2}$. Pick up $\gamma \in \in_{R} Z_{p}$ to compute $Y_{1}=Q^{\gamma}, Y=H^{\gamma}$. Choose a hash function $H_{1}:\{0,1\}^{*} \rightarrow\{0,1\}^{\lambda}$, then return $g p k=\left(p, G_{1}, G_{2}, G_{T}, e, P, Q, H, Y, Y_{1}, H_{1}\right)$ and the master key isk $=\gamma$.

(2) DKeyGen $(\boldsymbol{g p k}, \boldsymbol{j})$ : The area in which vehicles move is divided into $m$ regions. Each region corresponds to a unique region name $j, j \in\{1, \ldots, m\}$. TTP picks up $r \in_{R} Z_{p}^{*}$ and set $R L_{j}=\Phi$, then return $d p k_{j}=P^{\gamma}$ and $R L_{j}$ for region $j$. The $R S U$ s in region $j$ download and store the domain public key $d p k_{j}$. When a vehicle enters into this region, it will obtain the corresponding $d p k_{j}$ from $R S U s$. Considering the correlation attack, $d p k_{j}$ should be recomputed by TTP at regular intervals $\Delta T$.

(3)VKeyGen $(g p k, \gamma)$ : Before vehicle $V_{i}$ enters into any region, $V_{i}$ interacts with TTP to authenticate his identity and to obtain his/her long-term private key which remains the same for all periods.

- $V_{i}$ picks up $f^{\prime} \in_{R} Z_{p}$ and computes $F^{\prime}=Q^{f^{\prime}}$;

$\Pi=\operatorname{PoK}\left\{C=E x t-\operatorname{Commit}\left(f^{\prime}\right) \wedge N I Z K P E q D L\left(f^{\prime}, C, F^{\prime}, Q\right)\right\}$;

Send $F$ ' and $\Pi$ to TTP.

- TTP checks $\Pi$, and chooses $x, f^{\prime \prime} \in Z_{p}$, computes $F=F^{\prime} \cdot Q^{f^{\prime \prime}}, A=(P \cdot F)^{\frac{1}{\gamma+x}}, Z=e(A, H)$; Return $f^{\prime \prime}, A, x, Z$ to $V_{i}$.

- $V_{i}$ computes $f=f^{\prime}+f^{\prime \prime}$; And check $e\left(A, H^{x} \cdot Y\right) \stackrel{?}{=} e\left(P \cdot Q^{f}, H\right)$.

Finally, $V_{i}$ gets the long-term private key $u k_{i}=\left(f_{i}, A_{i}, x_{i}, Z_{i}\right)$; TTP maintains a tracking list $\boldsymbol{t} \mathbf{l}=\left(I D_{i}, A_{i}^{\gamma}\right)$ for dishonest vehicles, and a revoking token list $\boldsymbol{r t}_{\boldsymbol{i}}=(F, x)$ for pseudonym revocation.

(4) VDNymGen $\left(\boldsymbol{g p} \boldsymbol{k}, \boldsymbol{u s} \boldsymbol{k}_{\boldsymbol{i}}, \boldsymbol{d p} \boldsymbol{k}_{\boldsymbol{j}}\right)$ : When vehicle $V_{i}$ enters into a region $\boldsymbol{j}, V_{i}$ computes nym nij $_{i j}=$ $Q^{f_{i}} \cdot\left(d p k_{j}\right)^{x_{i}}$, which is the unique pseudonym of vehicle $V_{i}$ in the region $j$.

(5) $\operatorname{Sign}(g p k, u s k, d p k, n y m, m): V_{i}$ signs message $m$ in the following step:

- Choose $a, r_{a}, r_{f}, r_{x}, r_{b}, r_{d}, \in_{R} Z_{p}$, compute $T=A \cdot Q^{a}$ and $T_{t}=Y_{1}^{a}$;

- Set $R_{1}=H^{r_{f}} \cdot d p k^{r_{x}} ; R_{2}=n y m^{r_{a}} \cdot H^{-r_{d}} \cdot d p k^{-r_{b}} ; R_{3}=Z^{r_{x}} \cdot e(Q, H)^{a \cdot r_{x}-r_{f}-r_{b}} \cdot e(Q, Y)^{-r_{a}}$;

- Compute $c=H_{1}\left(d p k \|\right.$ nym $\left.\|T\| T_{t}\left\|R_{1}\right\| R_{2}\left\|R_{3}\right\| m\right)$;

- Set $s_{f}=r_{f}+c \cdot f ; s_{X}=r_{X}+c \cdot x ; s_{a}=r_{a}+c \cdot a ; s_{b}=r_{b}+c \cdot a \cdot x ; s_{d}=r_{d}+c \cdot a \cdot f$;

The signature of $m$ is $\sigma=\left(T, T_{t}, c, s_{f}, s_{x}, s_{a}, s_{b}, s_{d}\right)$.

(6) Verify $(g p k, d p k, n y m, \boldsymbol{m}, \sigma, \boldsymbol{R} L):$ If nym $\in R L$, reject the signature. Otherwise, the verifier computes $R_{1}^{\prime}=H^{s_{f}} \cdot d p k^{s_{x}} \cdot$ nym $^{-c} \quad ;$ $R_{2}^{\prime}=\operatorname{nym}^{s_{a}} \cdot H^{-s_{d}} \cdot d p k^{-s_{b}}$ $R_{3}^{\prime}=e(T, H)^{s_{x}} e(Q, H)^{-s_{f}-s_{b}} e(Q, Y)^{-s_{a}}\left[e(P, H) e(T, Y)^{-1}\right]^{-c}$; 
Compute $c^{\prime}=H_{1}\left(d p k\|n y m\| T\left\|T_{t}\right\| R_{1}^{\prime}\left\|R_{2}^{\prime}\right\| R_{3}^{\prime} \| m\right)$.

If $c=c^{\prime}$, accept it, otherwise return reject.

(7) DomainRevoke( $\left.g p k, d p k_{j}, r t_{i}, \boldsymbol{R} L_{j}\right)$ : If $V_{i}$ 's pseudonym of region $j$ needs to be revoked, RSUs will first obtain the revocation information, and transmit it to TTP. TTP parses $r t_{i}$ as $(F, x)$, and computes the revoked pseudonym as $\left\{a u x_{j}=F_{i} \cdot\left(d p k_{j}\right)^{x i}\right\}$, then returns the revoked list of region $j$ : $R L_{j}=R L_{j} \cup\left\{a u x_{j}\right\}$

(8) Tracking (gpk, isk, $\sigma, \boldsymbol{t l})$ : Once an accepted message $m$ of $V_{i}$ has been disputed, RSUs ask TTP for tracing the vehicle. TTP uses the master key $\gamma$ to compute

$T^{\gamma} / T_{t}=A_{i}^{\gamma} \cdot Q^{a \gamma} / Y_{1}^{a}=A_{i}^{\gamma} \cdot Q^{a \gamma} / Q^{a \gamma}=A_{i}^{\gamma}$

and can efficiently trace $V_{i}$ 's real identity $I D_{i}$ by looking up the tracking list $t=\left(I D_{i}, A_{i}^{\gamma}\right)$ he/she maintains.

\section{Security and Efficiency Analysis}

(1) Security analysis

Since the domain-specific pseudonym signature scheme ${ }^{[11]}$ has been proved to be seclusive, unforgeable and cross-domain anonymous in the random oracle under DL, DDH, SDH, q-SDH problem $^{[12]}$, the security of the proposed TRPS scheme can be guaranteed, i.e., it can effectively achieve cross-domain anonymity with conditional tracking to fulfill the requirements of location privacy. Considering no disputed messages in [11], the scheme has no Tracking algorithm. We add the tracking algorithm in our scheme, which is more practical in VANETs. The security of the algorithm is based on the DL assumption.

(2) Efficiency analysis

By using domain-specific pseudonym signature scheme, our scheme TRPS enables dynamic pseudonym generation without the self-certification. This alleviates the need for storing a set of certified pseudonyms before, which is a common solution to generate pseudonyms in the previous proposed schemes.

In addition, in the VKeyGen( $\cdot$ ) step, TTP computes the bilinear pairings and add it to vehicle's private key, this pre-computation help the vehicle avoid any pairing. In the signature step, the vehicle only needs to compute multi-exponentiations in $G_{1}$ and $G_{T}$, which help the vehicles to reduce computation cost.

\section{Conclusion}

In this paper, we propose a tracking-resistant pseudonym scheme (TRPS) for location privacy in VANETS. Our scheme supports efficient and unlinkable pseudonym changes as well as malicious pseudonym holders' revocation and trace. We analyze the security and efficiency of the proposed scheme. For future work, we intend to study the pseudonym changing strategy and the time interval.

\section{Acknowledgement}

The research was sponsored by the Fund Project of Domestic Visiting Scholars of Excellent Backbone Teachers of Higher Education Institutions in Shandong Province.

\section{References}

[1] D. Eckhoff, R. German, C. Sommer, et al. Slotswap: Strong and affordable location privacy in intelligent transportation systems, IEEE Communications Magazine, 2011, 49(11): 126-133.

[2] F. Dressler, F. Kargl, J. Ott, et al. Research challenges in inter-vehicular communication: lessons of the 2010 dagstuhl seminar, IEEE Communications Magazine, 2011, 49(5): 158-164. 
[3] M. Raya and J. Hubaux. Securing vehicular ad hoc networks, Journal of Computer Security, 2007, 15(1): 39-68.

[4] P. Papadimitratos, L. Buttyan, T. Holczer, et al. Secure vehicular communication systems: Design and architecture, IEEE Communications, 2008, 46(11): 100-109.

[5] A. Boualouache and S. Moussaoui. S2SI: A Practical Pseudonym Changing Strategy for Location Privacy in VANETs. 2014 International Conference on Advanced Networking Distributed Systems and Applications, 70-75.

[6] M. Florian, J. Walter, and I. Baumgart. Sybil-Resistant Pseudonymization and Pseudonym Change without Trusted Third Parties. WPES'15, 2015, 65-74.

[7] D. Förster , F. Kargl and H. Löhr. PUCA: A pseudonym scheme with strong privacy guarantees for vehicular ad-hoc networks. Ad Hoc Networks 2016, 37: 122-132.

[8] K. Moghraoui, B.A. Bensaber. An Efficient Pseudonym Change Protocol Based on Trusted Neighbours for Privacy and Anonymity in VANETs, DIVANet'15, 2015, ACM, 93-99.

[9] Y.Y. Pan, J.Q. Li, Cooperative pseudonym change scheme based on the number of neighbors in VANETs. Journal of Network and Computer Applications, 2013, 36: 1599-1609.

[10] B.Wiedersheim, ZD Ma, F. Kargl. Privacy in Inter-Vehicular Networks Why simple pseudonym change is not enough. The Seventh International Conference on Wireless On-demand Network Systems and Services, IEEE WONS 2010, 176-183.

[11] J. Bringer, H. Chabanne, R. Lescuyer. Efficient and Strongly Secure Dynamic DomainSpecific Pseudonymous Signatures for ID Documents[J]. Financial Cryptography and Data Security, 2014, LNCS 8437: 255-272.

[12] D. Boneh, X. Boyen, Short signatures without random oracles, the SDH assumption in bilinear groups, Journal of Crypt., 2008, 21(2):149-177. 